\title{
High Performance Computing with the Cell Broadband Engine
}

The Cell Broadband Engine was conceived to enable the design of novel and highly efficient systems for compute-intensive applications. The Cell/B.E. departs from prior architectures by adopting a heterogeneous chip multiprocessor architecture with novel accelerator cores and an explicitly managed memory hierarchy. The increased computing density of the design improves peak performance as well as power efficiency.

Beyond the Sony PlayStation 3, its first target system, the Cell Broadband Engine architecture has been used in systems ranging from compute-intensive embedded systems to high-end compute servers, demonstrating the versatility and power of its new concept. In 2008 the IBM RoadRunner supercomputer, incorporating nearly 13,000 Cell/B.E. processors, became the first system in the world to exceed the 1 Petaflop per second performance barrier as measured by the TOP500 list of the world's most powerful supercomputers. In the same year, Cell-based systems were also the world's most power-efficient systems as measured by the Green 500 list.

The Cell/B.E.'s departure from a traditional homogeneous SMP system design with hardware-managed caches change algorithms, programming models, compilers and run-time systems. The Cell/B.E. architecture offers new ways for software to control hardware resources that challenge software architects and algorithm researchers to rethink algorithms and computing paradigms to exploit its greatly increased performance potential.

Peak performance is obtained when vector operations are fully utilized in each accelerator core (SPE), all cores are simultaneously fully engaged, and by overlapping communication with computation using bulk DMA transfers between main memory and the SPEs. This benefits from a contiguous data layout of arrays as bulk DMA transfers are most efficient on contiguous data residing in memory. Such a layout for a 2-D array is square block format. This choice leads to very high Cell/B.E. performance of kernel routines that operate on these square block sub arrays simultaneously in the SPEs. In particular, this issue describes nearly perfectly performing matrix multiplication kernel routines. By exploiting these array layouts and overlapping Cell/B.E. bulk DMA transfers with computation to ensure that kernel routines will always have their new sub matrix operands in their SPE local stores ready for their next invocations, the Cell/B.E. usually reaches its full potential on the most important dense linear algebra algorithms.

The ultimate measure of the success of an architecture is the applications that it enables and the scientific breakthroughs that become possible with those applications. Thus, the spotlight in this issue is on innovative algorithms, programming models and application architectures to exploit this new hardware design.

The papers included in this special issue fall in three categories, covering performance engineering for key algorithms and library functions; programming models and runtime systems; and, most importantly, applications that build on these foundations to enable scientific discoveries across a wide range of disciplines.

We received 42 submissions from 16 countries in response to our call for papers. To select the best of these papers, we relied on an Editorial Board consisting of experts covering all areas of high-performance computing as well as on external subject matter experts. Together, they produced 138 reviews, or an average of over 3 reviews per paper. Based on these reviews, we selected twelve papers for this special issue.

In the performance engineering and library development category:

- Implementing a Parallel Matrix Factorization Library on the Cell Broadband Engine

- QR Factorization for the CELL Processor 
- Programming the Linpack Benchmark for the IBM PowerXCell 8i Processor

In the programming models and runtime systems category:

- Available Task-level Parallelism on the Cell/B.E.

- CellSs: scheduling techniques to better exploit memory hierarchy

In the applications category:

- High Performance Protein Sequence Database Scanning on the Cell/B.E. Processor

- Building High-Resolution Sky Images using the Cell/B.E.

- Implementation of scientific computing applications on the Cell Broadband Engine

- Efficient SIMDization and Data Management of the Lattice QCD Computation on the Cell Broadband Engine

- Streaming Model Based Volume Ray Casting Implementation for Cell Broadband Engine

- 3D Seismic Imaging through Reverse-Time Migration on Homogeneous and Heterogeneous Multi-core Processors
- Implementation and Performance Modeling of Deterministic Particle Transport (Sweep3d) on the IBM Cell/B.E.

We want to thank all authors for their excellent contributions, only a few of which we were able to accommodate in this special issue. We would like to thank the members of the Editorial Board and external experts who guided us in the selection of these papers for their many excellent reviews, expert suggestions and insights.

Finally, we want to thank the Editor-in-Chief of Scientific Programming, Prof. Boleslaw Szymanski, who guided it us through the entire process of preparing the issue with encouragement and expert advice.

We hope you have as much enjoyment in reading this issue as we had in preparing it!

\author{
Michael Gschwind \\ Fred Gustavson \\ Jan F. Prins
}

December 2008 

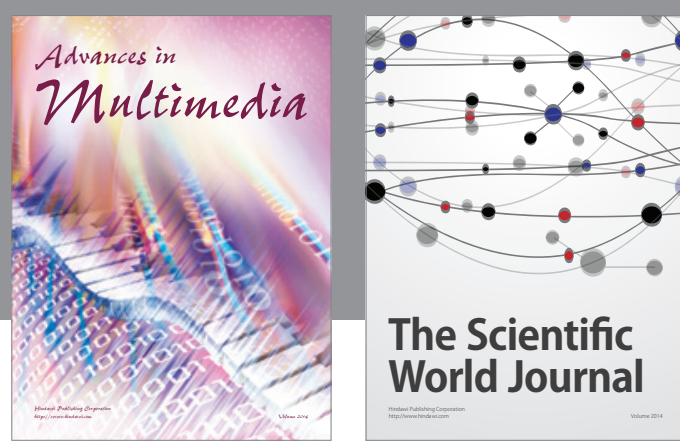

The Scientific World Journal
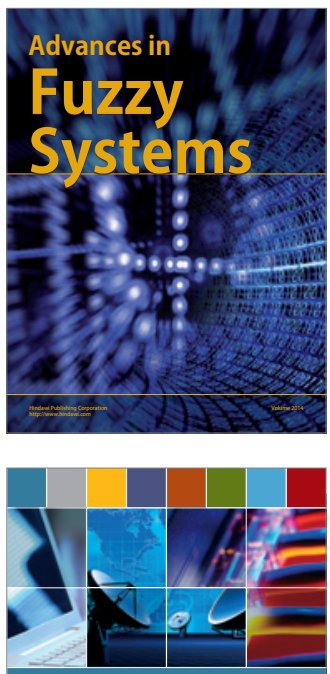

Computer Networks and Communications
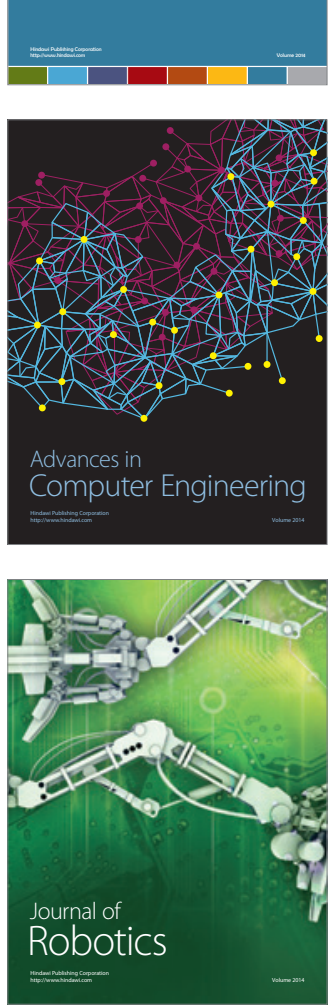
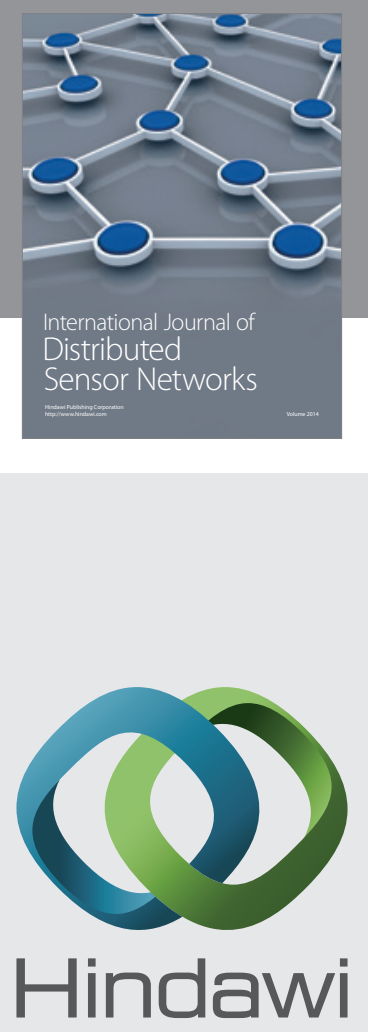

Submit your manuscripts at

http://www.hindawi.com
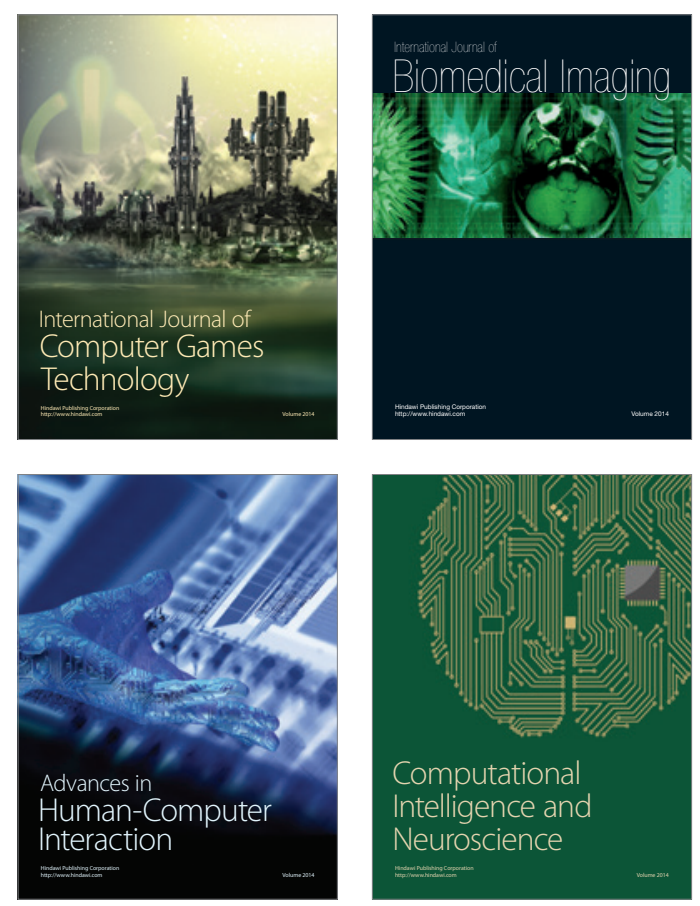
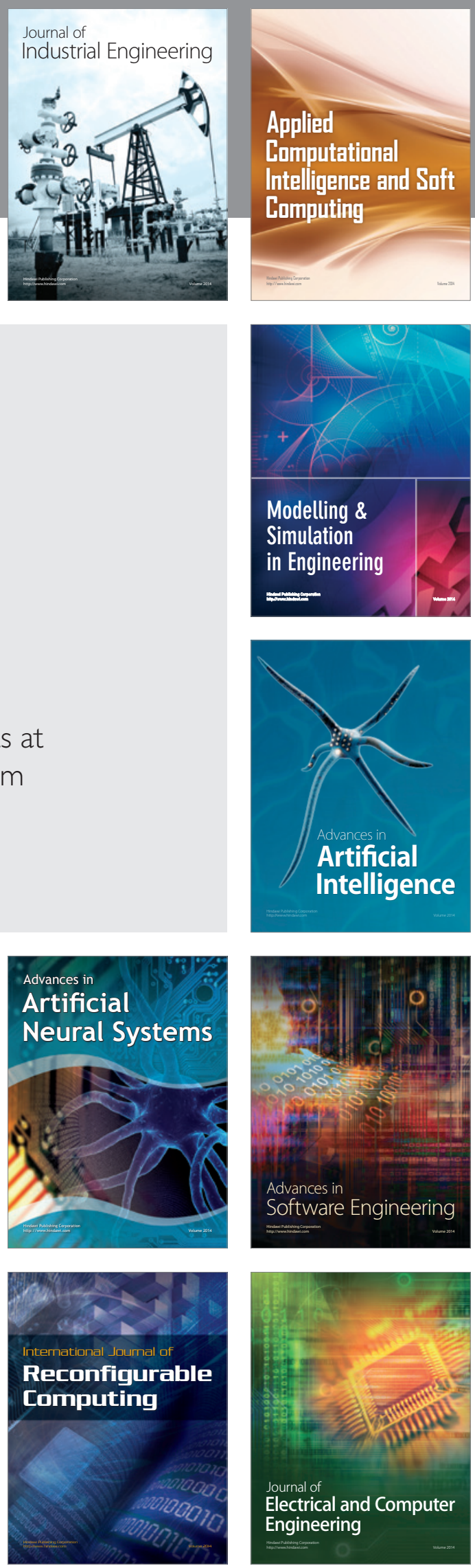